\title{
Gamma Knife Radiosurgery for Berry Aneurysms: Quo Vadis
}

\author{
Manjul Tripathi'^ Aman Batish ${ }^{2}$ Sandeep Mohindra ${ }^{3}$
}

${ }^{1}$ Department of Neurosurgery, Postgraduate Institute of Medical Education and Research, Chandigarh, India
Address for Correspondence Manjul Tripathi, MCh, Department of Neurosurgery, Neurosurgery Office, Nehru Hospital, 5th Floor, Post Graduate Institute of Medical Education \& Research, Chandigarh 160012, India (e-mail: drmanjultripathi@gmail.com).

\begin{abstract}
Keywords

- aneurysm

- arteriovenous

malformation

- fistula

- cavernoma

- radiation

- radiosurgery

Background Intracranial aneurysms are vascular malformations with significant mortality and morbidity profile. Various treatment modalities have been developed to positively impact the outcome profile with gradual shift to the minimally invasive treatment modalities. Gamma knife radiosurgery (GKRS) is an established primary treatment modality for various intracranial arteriovenous malformations (AVMs); however, its efficacy for berry aneurysmal obliteration has been historically dismal.

Objective The aim of this study is to evaluate the factors responsible for poor radio surgical outcome for intracranial aneurysms.

Methods The literature is reviewed for the differential efficacy of GKRS for aneurysm and AVM.

Results Though both are vascular malformations, aneurysm and AVM have inherent differences in angioarchitecture, intracranial location, surrounding neighborhood, radio-sensitivity, and latency for obliteration. The major difference arises because of surrounding neighborhood of connective tissue stroma which stabilizes the irradiated pathology.

Conclusion Though considered radioresistant, aneurysms show promising results with animal models of radiosurgery. The future lies in two hypothetical improvements: with a supporting neighborhood or sensitization of the vessel wall that may change the natural history of an aneurysm, especially an unruptured one.
\end{abstract}

In our quest to make treatments better, safer, acceptable, and minimally invasive to the patients, gamma knife radiosurgery (GKRS) has made a paradigm shift in the management of various intracranial malformations, especially arteriovenous malformations (AVM). ${ }^{1}$ After its efficacy in successful obliteration of AVM nidus by Steiner et al in 1972 and later disappearance of intranidal aneurysms with nidus obliteration, GKRS is viewed as a potential treatment option for aneurysmal obliteration. ${ }^{2}$ Aneurysms associated with AVM show occlusion in nearly $71 \%$ of the patients (especially if they are flow-related intranidal or postnidal aneurysms), but this happens because of AVM obliteration and flow reduction. ${ }^{3,4}$ The hypothetical goals of GKRS for aneurysm are volumetric reduction and wall thickening to obliterate it. The results were disappointing for GKRS for berry aneurysms. It is high time to understand the differential efficacy of GKRS for berry aneurysms and the future directions for a research on the same.
DOI https://doi.org/ 10.1055/s-0040-1716795 ISSN 0976-3147.
(C) 2020. Association for Helping Neurosurgical Sick People.

This is an open access article published by Thieme under the terms of the Creative Commons Attribution-NonDerivative-NonCommercial-License, permitting copying and reproduction so long as the original work is given appropriate credit. Contents may not be used for commercial purposes, or adapted, remixed, transformed or built upon. (https://creativecommons.org/licenses/by-nc-nd/4.0/)

Thieme Medical and Scientific Publishers Pvt. Ltd., A-12, 2nd Floor, Sector 2, Noida-201301 UP, India 


\section{Histopathological Changes after Gamma Knife Radiosurgery of Arteriovenous Malformation}

The autopsy specimen of irradiated AVM has revealed different stages of tissue reactions that include endothelial destruction, granulation tissue formation, followed by spindle cell infiltration, scar tissue replacement, and hyaline degeneration. Schneider et al found expansion of extracellular matrix with hyaline and calcium deposition and hypertrophy of collage type $\mathrm{V}$ to consolidate the obliterative process. In completely obliterated AVMs, the nidus is replaced by hyaline scar and only vessels contour is recognizable without any identifiable lumen. The incompletely obliterated AVMs have lumen obstructed by fibrin thrombi ( - Fig. 1). ${ }^{5}$

\section{Histopathological Changes after Gamma Knife Radiosurgery of Aneurysm}

Animal model studies have created elastase induced model in rabbit carotid artery. ${ }^{6}$ Their stability has already been proven by long-term patency and maintenance of dimension without rupture. ${ }^{7}$ In a rabbit model, Meadowcroft et al treated aneurysm with a conformal radiation dose of $25 \mathrm{~Gy}$ at $50 \%$ isodose with GKRS and followed these aneurysms on longitudinal magnetic resonance imaging studies for 2 years tracking their size and shape index modifications. Finally, aneurysms were sacrificed for histopathological evaluations. Histopathologic review (HPR) evaluations showed progressive reduction in size ( $1.7 \%$ per month), wall thickening $(0.3 \%$ per month) with subintimal necrosis. No aneurysm ruptured during the observation period. Consistent shape indices (nonsphericity of the average volume, aspect ratio, and isoperimetric ratio of whole aneurysm volume) demonstrated stable aneurysm patency and maintenance of minimal rupture risk during the treatment. ${ }^{8}$

\section{How Is It Different for Arteriovenous Malformation and Aneurysm?}

Apart from the difference in the vessel wall, the differential outcome is predominantly decided by the neighborhood of the pathology. AVMs are intraparenchymal structures while aneurysm bathe in cerebrospinal fluid. The connective tissue stroma surrounding an AVM is rich in spindle-shaped cells and myofibroblast. Radiosurgery causes rapid activation of myofibroblast in the connective tissue stroma and helps in the hyalinization of the occluded vessels periphery. It is the hyalinization of the scar tissue which stabilizes the obstructed AVM vessels once they get occluded. An aneurysm lacks that neighborhood of connective tissue stroma that could stabilize a vessel wall undergoing changes with radiosurgery. This makes other vascular pathologies relatively resistant to the radiosurgery as cavernomas are usually surrounded by hemosiderin ring and fistulas also similar surroundings. ${ }^{8}$

\section{Can We Irradiate Incidental Aneurysms?}

All treatments for aneurysm occlusion are invasive to variable extent; hence, no consensus guideline could be drawn for incidental aneurysms. Many times, patients agree to opt for watchful observation with all the inherent risks known. If found successful, GKRS may be a noninvasive option for incidental unruptured intracranial aneurysms. In view of differential natural history and mortality profile of an incidental aneurysm with AVM, it remains imperative to analyze the obliteration rate of an aneurysm after radiosurgery. Stereotactic radiosurgery (SRS) reduces the risk of AVM hemorrhage, which is indirectly driven by factors such as marginal dose, location, size, associated anomalies (such as aneurysms and fistula), etc. Using the hemorrhage rate of 2 to $4 \%$, there does not seem to be significant change of hemorrhage rate following SRS until the AVM is completely

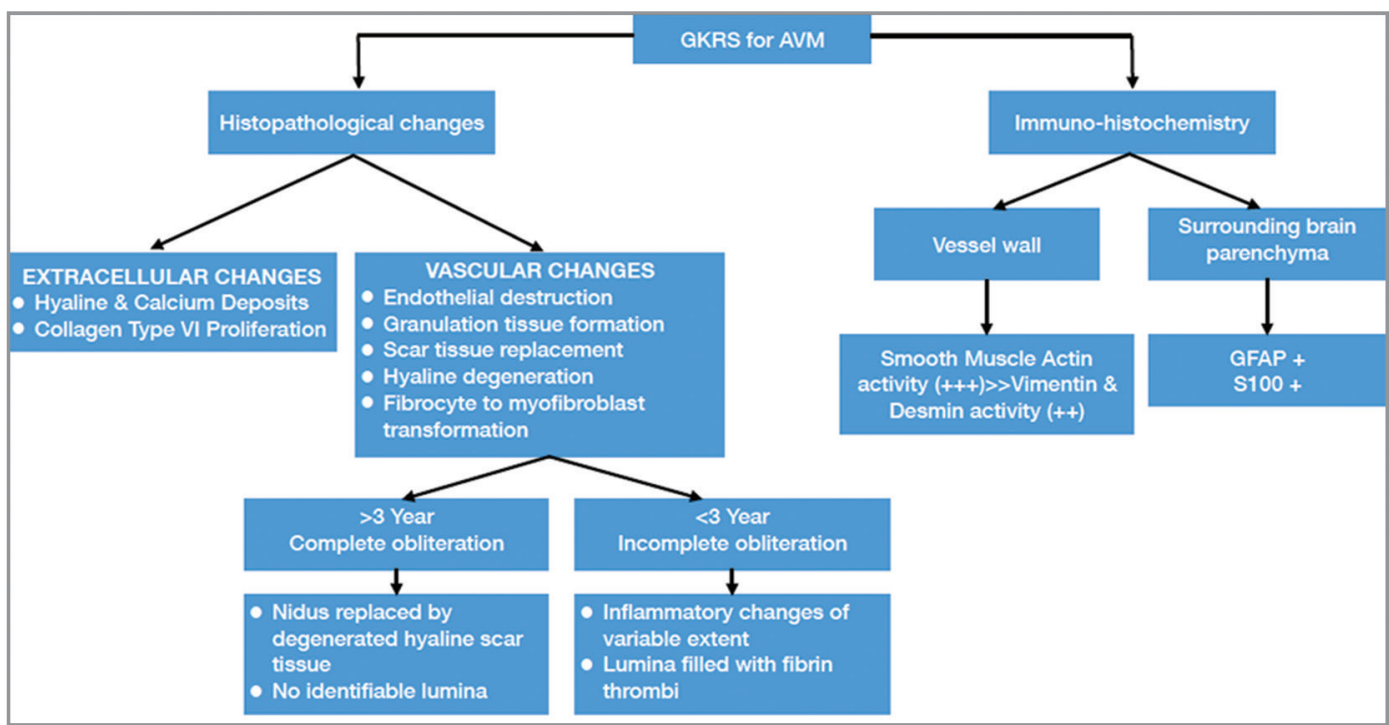

Fig. 1 Morphological changes in arteriovenous malformation after gamma knife radiosurgery. AVM, arteriovenous malformations; GFAP, glial fibrillary acidic protein; GKRS, gamma knife radiosurgery. 
obliterated. ${ }^{9}$ Theoretically, the latency period for aneurysm obliteration must be higher than an AVM. An important consideration should be given to a possibility of wall thinning after radiosurgery and iatrogenic pseudo aneurysm formation. There are published reports of pseudo-aneurysm formation in superior cerebellar artery (SCA) and anterior inferior cerebellar artery in cases of trigeminal neuralgia, and meningioma of cerebellopontine angle. ${ }^{10-14}$ Another patient developed a pseudoaneurysm in petrous segment of internal carotid artery, following GKRS for pituitary adenoma (PA). This patient needed high-flow bypass and trapping of aneurysm and obtained good recoverey. ${ }^{15}$ With GKRS, this possibility remains higher than other radio surgical tools as gamma knife is a heterogeneous dose distribution, and a hotspot on the vessel wall may cause focal elastin degeneration and wall weakening.

\section{Conclusion}

Results of GKRS for berry aneurysms may be improved by two hypothetical improvements: with a supporting neighborhood or sensitization of the vessel wall. A subarachnoid infiltration of myofibroblast rich medium in the vicinity of aneurysm may help in the radiation induced fibrosis further supporting intraluminal inflammation. A sensitizing agent to irradiation may also promote saccular wall thickening and lumen occlusion. Future laboratory-based studies are warranted to solicit conclusive answers.

\section{Key Message}

Gamma knife radiosurgery leads to vascular wall thickening, histopathological changes, and linear volumetric reduction of the vascular lumen. The result of radiosurgery in an aneurysm is not as robust as for an arteriovenous malformation. Gamma knife radiosurgery remains a futuristic viable, minimally invasive option for unruptured intracranial saccular aneurysm.

\section{Funding \\ None.}

\section{Conflict of Interest}

None declared.

\section{Acknowledgments}

All authors are sincerely thankful to late Prof. Kanchan Kumar Mukherjee for his vision and thought-provoking discussions on this topic. They also express their sincere thanks to department of Radiotherapy, PGIMER Chandigarh for the valuable support.

\section{References}

1 Mukherjee KK, Kumar N, Tripathi M, et al. Dose fractionated gamma knife radiosurgery for large arteriovenous malformations on daily or alternate day schedule outside the linear quadratic model: proof of concept and early results. A substitute to volume fractionation. Neurol India 2017;65(4):826-835

2 Steiner L, Leksell L, Greitz T, Forster DM, Backlund EO. Stereotaxic radiosurgery for cerebral arteriovenous malformations. Report of a case. Acta Chir Scand 1972;138(5):459-464

3 Szeifert GT, Levivier M, Lorenzoni J, Nary I, Major O, Kemeny AA, Morphological observations in brain arteriovenous malformations after gamma knife radiosurgery. In: Niranjan A, Kano H, Lunsford LD, eds. Gamma Knife Radiosurgery for Brain Vascular Malformations. Prog Neurol Surg. Basel, Karger; $2013 ; 27: 119-229$

4 Tripathi M. Letter to the Editor. Dose-fractionated gamma knife radiosurgery for large-volume arteriovenous malformations. J Neurosurg 2018;129(6):1660-1662

5 Schneider BF, Eberhard DA, Steiner LE. Histopathology of arteriovenous malformations after gamma knife radiosurgery. J Neurosurg 1997;87(3):352-357

6 Altes TA, Cloft HJ, Short JG, et al; American Roentgen Ray Society. 1999 ARRS Executive Council Award. Creation of saccular aneurysms in the rabbit: a model suitable for testing endovascular devices. AJR Am J Roentgenol 2000;174(2):349-354

7 Ding YH, Dai D, Lewis DA, et al. Long-term patency of elastase-induced aneurysm model in rabbits. AJNR Am J Neuroradiol 2006;27(1):139-141

8 Meadowcroft MD, Cooper TK, Rupprecht S, et al. Gamma Knife radiosurgery of saccular aneurysms in a rabbit model. J Neurosurg 2018;129(6):1530-1540

9 Ding D, Chen CJ, Starke RM, et al. Risk of brain arteriovenous malformation hemorrhage before and after stereotactic radiosurgery. Stroke 2019;50(6):1384-1391

10 Chen JCT, Chao K, Rahimian J. De novo superior cerebellar artery aneurysm following radiosurgery for trigeminal neuralgia. J Clin Neurosci 2017;38:87-90

11 Endo $\mathrm{H}$, Fujimura M, Inoue T, et al. Simultaneous occurrence of subarachnoid hemorrhage and epistaxis due to ruptured petrous internal carotid artery aneurysm: association with transsphenoidal surgery and radiation therapy: case report. Neurol Med Chir (Tokyo) 2011;51(3):226-229

12 Kellner CP, McDowell MM, Connolly ES Jr, Sisti MB, Lavine SD. Late onset aneurysm development following radiosurgical obliteration of a cerebellopontine angle meningioma. J Neurointerv Surg 2015;7(6):e21

13 Uchikawa H, Nishi T, Kaku Y, Goto T, Kuratsu JI, Yano S. Delayed development of aneurysms following gamma knife surgery for trigeminal neuralgia: report of 2 cases. World Neurosurg 2017;99:813.e13-813.e19

14 Akai T, Torigoe K, Fukushima M, Iizuka H, Hayashi Y. De novo aneurysm formation following gamma knife surgery for arteriovenous malformation: a case report. J Neurol Surg Rep 2015;76(1):e105-e108

15 Inoue $\mathrm{H}$, Kawano $\mathrm{T}$, Ohmori $\mathrm{Y}$, et al. Internal carotid artery aneurysms diagnosed after stereotactic radiosurgery for a growth hormone-secreting pituitary adenoma: a case report and literature review. Acta Neurochir (Wien) 2019;161(6):1191-1195 